\title{
Several Theoretical Issues Worthy of Attention in Psychological Counseling in Colleges
}

\author{
Shulian Li \\ Jilin Normal University, Siping City, Jilin Province, 136000 \\ mailbox: 1shlja@126.com
}

\begin{abstract}
$\overline{\text { Abstract:With the rapid development of society and the continuous deepening of reform and opening up, the current }}$ psychological pressure on college students is getting worse. Psychological counseling in colleges and universities is an important measure to improve the psychological quality of college students and maintain and enhance the mental health of college students. Under the current situation, it has gradually attracted the attention of all sectors of society. This article briefly expounds the significance and development ideas of college psychological counseling work, analyzes and discusses several issues worth noting in the process of psychological counseling work, and provides references for promoting the continuous development of college psychological counseling work.
\end{abstract}

Keywords:Colleges and Universities; College Students; Psychological Counseling; Theoretical Issues

In recent years, the work of psychological counseling in universities in my country has developed rapidly. Many universities have included psychological counseling in students' ideological education or medical care. This shows that the mental health of college students has received widespread attention from all walks of life. . Strengthen the mental health education of college students and promote the development of scientific and standardized psychological counseling work in colleges and universities ${ }^{[1]}$ Although the psychological counseling work seems to be carried out smoothly, judging from the actual situation of various universities, there are still many problems, such as differences in development caused by different regions, weak professional strength despite active participation, and psychological problems in some universities. There is still an initial stage in the development of consulting work, and there is no rich practical experience. It needs further research and discussion to solve it.

\section{The significance of psychological counseling in universities}

\subsection{Psychological consultation in colleges is an inevitable requirement of quality education}

Quality education puts forward four requirements for the improvement of students' quality, namely physiology, culture, morality and psychology. As the foundation and core of quality education, the improvement of psychological quality is an important task for contemporary college students to achieve quality education. At present, the state strongly advocates the transition from test-oriented education to quality education. The improvement of students' quality is not limited to cultural and scientific knowledge. The acquisition of labor skills, the cultivation of moral quality and the maintenance of mental health are the ultimate goals of quality education. Psychological counseling is a specific measure for colleges and universities to implement mental health education and guidance for students, and it is also the only measure to improve psychological quality. Therefore, the development of psychological counseling work is not only the development demand of college mental health guidance, but also the inevitable requirement of national quality education.

1.2 Psychological consultation in colleges is a necessary demand for the psychological growth Copyright $(92020$ Shulian Li

doi: 10.18282/le.v9i7.1529

This is an open-access article distributed under the terms of the Creative Commons Attribution Non-Commercial License

(http://creativecommons.org/licenses/by-nc/4.0/), which permits unrestricted non-commercial use, distribution, and reproduction in any medium, provided the original work is properly cited. 


\section{of college students}

With the rapid development of society and economy, people's pace of life is accelerating. Affected by the rapid changes in the times, contemporary college students are facing increasing pressures on factors such as study and life. In addition, some colleges and universities ignore students' mental health problems. Health is not guaranteed. The development of psychological counseling in colleges and universities can effectively solve the common problems of contemporary college students in learning, interpersonal relationships, sexual physiological and psychological confusion, psychological confusion in life, pathological problems caused by psychological disorders, etc., and promote the mental health of college students.

\section{Ideas for the development of psychological counseling in universities}

\subsection{Close cooperation between psychological counseling and moral education}

Psychological consultation is the education and guidance work carried out to adjust the psychological problems of students such as psychological deviation, psychological imbalance, interpersonal relationship, and maintain a healthy psychological state. The content of moral education includes: students' mental health knowledge, moral quality, psychological debugging ability, value cultivation and so on. Although the work content of the two is different, they are closely related. Psychological counseling work can be closely coordinated with moral education work to give play to their respective work effects, and explore the best educational methods and channels for students' psychological problems. The mutual cooperation of psychological consultation and moral education can effectively improve the effectiveness of the two.

\subsection{Multi-level and diversity}

There are individual differences in the level of students' psychological development, and the causes of students' psychological problems are diverse. Faced with different individual needs, different consulting models and methods should be used to reflect the multi-level and diversity of college psychological consulting work.

When student development is used as the content of mental health education, it is oriented to the group for all students, and it can also be open through classroom lectures, group counseling, mental health lectures, posters, and broadcasting. For the prevention of psychological problems of some students, it is necessary to be proactive and foreseeable, to provide psychological counseling to the group of students in the high-incidence period of psychological problems, to timely allocate psychological obstacles, and to enhance the students' self-regulation and self-control ability. When a small number of students have psychological problems, targeted guidance can be used to ease or eliminate students' bad emotions due to psychological problems through one-to-one consultation services. The work of psychological counseling focuses on the prevention and intervention of the mental health of all students, coordinating the relationship between the individual and the whole, can effectively improve the efficiency of psychological counseling.

\section{Problems that should be paid attention to in the development of psychological consultation in colleges}

\subsection{The establishment of core concepts of developmental psychological consultation}

College psychological counseling mainly includes obstacle counseling and developmental counseling. ${ }^{[1]}$ Barrier psychological counseling usually refers to providing assistance and correction to students with psychological barriers, and its work content and form tend to be psychotherapy; developmental psychological counseling is based on the physical and mental development characteristics of students at different ages. Guide work for the purpose of inner contradiction, full understanding of self, and promotion of personality development. The current forms of psychological counseling in colleges and universities tend to be more in the category of psychotherapy. ${ }^{[2]}$

Indeed, some students in colleges and universities have different degrees of psychological barriers, so it is correct to start with psychological barriers in the development of college psychological counseling. However, for the vast majority of college students, there are only a small number of students who have serious psychological barriers and constitute 
mental illness, and more students are faced with psychological puzzles caused by factors such as daily life, study, and interpersonal communication. Counseling for some groups of psychological confusion, psychological treatment methods have great limitations. Developmental psychological counseling is proactive and forward-looking. It can effectively provide timely counseling and intervention for most students with psychological confusion, effectively prevent the process of students' psychological problems from small to large, and promote the healthy development of students' body and mind. Help students perfect the shaping of their personality. Therefore, the current college psychological counseling service should establish a concept with developmental psychological counseling as the core.

\subsection{Correct understanding of mental health standards}

Human psychology is a dynamic composition with complex structure. Mental health is not a simple matter of black and white. Students' mental health status should be viewed with a developmental perspective. From healthy to unhealthy, there is a slowly changing transition zone. Most students' psychological conditions are in this transition zone, which can be understood as psychological Sub-health. Human psychology is complex and changeable, and it can present different appearances with changes in times, social development, regional differences and other issues. Therefore, mental health standards should also be a dynamic and unquantifiable concept, which should fully integrate subjective and objective Combine standards. In the development of psychological counseling work in colleges and universities, attention should be paid to distinguishing the essence of students' abnormal behaviors, whether it is a short-term stress response or personality traits. University survival in a moderately stressed state is normal, and it is essentially different from pathological personality. . In addition, it is necessary to pay attention to distinguish between growth psychological changes and psychological abnormalities. People have corresponding psychological characteristics at different ages, such as adolescent rebellion and precocious puberty, which are all normal psychological categories and cannot be treated as psychological abnormalities. Its seriousness. A mentally healthy person's behavior and habits are compatible with his age, status, and social role, and his social environment is in harmony, and vice versa. ${ }^{[3]}$

\subsection{Treat psychological tests correctly}

Psychological tests are an important part of psychological diagnosis in the psychological counseling work of schools, and help to correctly distinguish the nature of students' psychological problems. Although psychological tests have a certain scientific basis, they cannot be overly dependent on the test results, and the results cannot be used to judge a person's mental health. As a tool for psychological diagnosis, psychological tests have imperfect theories and methods. There are many factors that affect test results, and the quantitative results of tests cannot be used as accurate indicators. Therefore, many psychologists currently advocate the use of psychological testing as an auxiliary tool for diagnosis, combining the test results with other methods, and conducting comprehensive analysis from the individual's comprehensive situation, so that the diagnostic results obtained are relatively reliable.

The preparation and selection of psychological tests need to be carefully considered. For the preparation of psychological tests, we must first have a profound theoretical basis of psychology and follow the principles of scientific writing. Test personnel should have good professional quality to avoid errors in the results caused by omissions in the test process. The test process should be kept confidential, exclude all factors that may affect the test results, and ensure the accuracy of the test results. Psychological test is an important means of psychological diagnosis, but it is not necessary. Before conducting a psychological test, it is necessary to make a preliminary judgment on the psychological state of the counseling object through observation, conversation, etc., and then decide whether to perform the test or which test to choose.

\subsection{Network consultation}

With the current popularization of the Internet in colleges and universities, online consultation has become a new channel for colleges and universities to carry out psychological consultation. Contemporary college students are already very proficient in using the Internet. Compared with face-to-face consultation, online consultation is more convenient, faster, and has higher privacy. For some people with psychological confusion, psychological obstacles and unwillingness to directly accept the psychological For tutoring students, online counseling is the best choice for psychological counseling. 
Currently, online consultation is mainly carried out in three forms: email, social software, and online meeting. Among them, e-mail and social software are frequently used. E-mail is regarded as an extension of letters. The consultant can make preliminary tentative judgments through the analysis of the content of the e-mail. The exchange of e-mails is the process of diagnosis and treatment. For minor students, the effect is good. Consultation conducted through social software can be regarded as a real-time question and answer process, which can understand the current psychological state of the counselor in time, and provide corresponding advice and guidance. Online meetings are usually aimed at large groups with a large number of people, and are almost the same as collective teaching on campus. They only break through the limitations of space in the form of consultation.

Although online consultation has certain convenience, it lacks the transmission of key information such as language and emotions between people, which leads to a decrease in the accuracy of judging the mental state of students. In the development of psychological counseling, face-to-face emotional expression and emotional performance are sometimes more accurate than the information conveyed by language. It can be seen that online consultation has certain convenience, but there are also certain limitations.

\section{In conclusion}

Psychological counseling is a key part of the mental health education in colleges and universities. Although it is carried out in most colleges and universities, there are still many problems in the actual psychological counseling work. Therefore, colleges and universities should put forward corresponding improvement measures for the various problems faced by the current psychological counseling work, establish perfect psychological counseling services, and provide effective guarantees for the mental health and personality improvement of college students.

\section{References}

1. Cui Jinggui. Analysis on the development trend of psychological counseling in my country's universities [J]. Design Art Research, 1998(3): 27-29.

2. Lu Aixin. Several noteworthy issues in psychological counseling in colleges and universities[J]. Journal of Luoyang Technical College, 2004.

3. Ma Yanxiang. On the Mental Health and Education of College Students_— The Second Study of "Mental Health Education: The Way to Perfect the Personality of College Students”[J]. Social Sciences, 2005(05):194-196. 